\title{
Nanotechnologies in the health care system
}

\begin{abstract}
Commercialization of nanotechnologies in Russian Health Care System (RHCS) requires detailed analysis of existing obstacles to technology translation. Here we will discuss three key innovations (i) Prof. Ilizarov's apparatus, (ii) 'Perftorun', known as 'blue blood' therapy of Russian prof. Beloyartsev, (iii) 'Litar' and artificial bone technology invented by Prof. Krasnov which is used to replace bones defects. We will consider challenges of Russian bionanotechnology clusters and education of scientists concerning the principles of technology transfer. Prof. Petrov, a coauthor of this paper, has extensive experience in implementation of novel technologies for health protection and safety. Historic data and case studies suggest that Russia's technology innovations require 30 - 40 years before it is successfully commercialization compared to 5 to 10 years in the United States. Substantial investment capital significant and high probability of technology failure in preclinical or clinical trials hinder commercialization of biomedical and health technologies in developed countries. Stringent regulatory approval process further increases the time and cost of moving the technology from laboratory into commercialization. Patent protection of new inventions is a key strategy for attracting substantial investment required for early stage transition of biomedical technologies into commercial products. Following the discussion of the three innovations mentioned above, our paper will suggest approaches of how to enhance commercial translation.
\end{abstract}

Keywords: technology translation, biomedical innovation, commercialization, Russian health care system, nanotechnologies
Volume 6 Issue I - 2020

\author{
Petrov Sergey, ${ }^{\prime}$ Valyaev Alexandr ${ }^{2}$ \\ 'Moscow State Pedagogical University, Russia \\ ${ }^{2}$ Nuclear Safety Institute RAS, Russia
}

Correspondence: Petrov Sergey, Moscow State Pedagogical University, I/I M. Pirogovskaya Str., Moscow, Russia, Tel +7(495) 315 83 72, Email svpetro47@mail.ru

Received: December 13, 2019 | Published: February 10, 2020

\section{Introduction}

Nanotechnology is science, engineering, and technology conducted at nanoscale of 1 - 100 nanometers. Nanoscale devices and systems are actively researched around the world and many have been commercialized. Nevertheless, the use of nanotechnology has not yet reached its full potential in medicine. Accelerating innovation in Russia's health care system would require detailed analysis of a wide range of challenges that hindered commercialization over the past several decades. Possible solutions to existing obstacles have to be explored and evaluated based on complex interplay of health policy and technology development as well as financial, corporate and legal system. To authors' knowledge, existing practices and solutions don't take into account significant issues that are detrimental to successful commercialization of biomedical innovation. Because obstacles to commercialization are not timely identified many important medical technology developments are either slowed or become terminated. In 2008, the Russian Government adopted "The Nanoindustry Development Program in the Russian Federation (RF) until 2015," the program led by Rosnano which by 2015 aimed to generate more than 900 billion Rubles from manufacturing of nanotechnology products. The Rosnano Supervisory Council approved the financing of 93 projects with the total budget of 300 billion rubles. The projects were developed across 6 technology clusters: energy saving; nanostructured materials; medicine and biotechnology; optoelectronics, nanoelectronics and others. The wide research and translation of nanotechnologies in medicine began in the following areas:
I. membrane plasmapheresis apparatus production;
II. micro-sources and microspheres for brachytherapy;

III. vaccines based on viral nanoparticles;

IV. antibiotics extracted from bacteria and microalgae;

$\mathrm{V}$. the use of cellular stress nanomodulators for the treatment of multiple sclerosis, infectious diseases and cancer;

VI. nanofilm bioactivated small-size biosensors;

VII. restoration of lost bones, teeth, cartilage and liver treatment.

Many new educational and research disciplines were opened in Russia's universities. However, the increase in number of graduates of these new programs did not appear to improve the quality or suitability of these graduates to high-technology industries. Unfortunately, significant investment allocated by Rosnano led to only very few successful technology developments. Many Rosnano projects were closed and substantial funding was used inappropriately as evidenced by many prior publications: http://www.yuri-kuzovkov. $\mathrm{ru} /$ journalism/corruption_russia/43_rosnano/, http://www.pppnr.ru/ news/-rosnano----apogej-rossijskoj-korruptsii/, http://korrossia.ru/ russia/mow/5571-rosnano-dlya-korrupcii.html Here we attempt to explain some reasons which may have led to high failure rate and inefficient capital investment into Russia's nanotechnology projects. We will briefly consider existing obstacles that hinder technology transfer and commercialization, particularly in RHCS. With the aim to describe issues that impede development of new technologies we present several examples of typical problems.

\section{Example I}

Developed in 1950 at an Institute of the Russia's Kurgan city, Professor Gavriil Ilizarov's apparatus has been used in treatment of open and fragmentation fractures. The peculiar fact is that Prof. 
Ilizarov was appointed as the head of a laboratory which implemented the proposed method only in 1966 i.e., more than 15 years after his invention. A significant clinical milestone occurred in 1968, when Ilizarov's apparatus was successfully used to heal multiple leg fractures of Olympic's jumping champion Valery Brumel. His book describes details of his miracle recovery following the use of Ilizarov's apparatus. Although "Nanotechnologies" are never mentioned in this book, there is opportunity for combining nanotechnologies with the Ilizarov's apparatus to enhance fracture healing process. Medical Plastik, an Italian company, greatly contributed to the rapid expansion of Ilizarov's method across the world. As a result, patients in other countries had benefited from this technology before Russia's health officials recognized the potential of Ilizarov's apparatus. Many lives and our country's prestige were lost from country's lack of support to this breakthrough medical technology. Only after 30 years from the time of Ilizarov's first experiment, the Kurgan Institute was recognized as the major scientific center and opened its branches in 10 other USRR cities. Ultimately Ilizarov had overcame all the bureaucratic barriers, but died of heart failure in 1992, the time when he was making fast progress in his technology's commercialization.

\section{Example 2}

The drug Perftorun ("blue blood") which was invented by USSR's professor Felix Beloyartsev (1941-1985) at the Institute of Biological Physics of the USSR Academy of Sciences. The drug successfully addressed the blood saving problems during numerous surgical procedures in military medicine and in the treatment of injured miners. Although this certified technology was recognized with the many awards, it did not receive support from USSR's health officials. Those who are interested in learning more about Perftorun's history can find additional information in the Internet. Failing to receive support, in 1985, professor Beloyartsev committed a suicide. Dr. Henrik Ivanitsky continued this work. The hard burden stemming from total suspicion and searching for enemies brought many negative consequences to the USSR society and science. Prof. Andrei Sakharov was turned from a homeland patriot and the USSR Hero of Labor into a dissident. Our homeland officials could not accept Sakharov's well-thought proposals that he made and put before these officials. The history of the breakdown of outdated government's apparatus was described by our famous writers including Nikolai Gogol, Anton Chekhov, Mikhail Bulgakov, Valdimir Mayakovsky, Eugenie Yevtushenko. The USSR's officials' incompetence in the field of science has led to immigration of our scientists and ideas to foreign countries. The weakening of Russia's scientific potential acquired increasingly sophisticated forms of brains drain through mandatory publications in the world international journals that is beneficial for our competitors. This is because publications of new technologies in scientific literature without proper intellectual protection result in lost opportunities for commercialization.

\section{Example 3}

Invented by professors Alexander Krasnov \& Sergei Litvinov from Russia's Samara city, the "artificial bone" is the technology to replace bones and other tissues. This technology, known as LitAr, has a 30-year history. The invention resulted in the creation of LitAr implantable material that can inhibit or prevent bone resorption and reduce scarring from surgical procedures. ${ }^{2}$ The material looks like a loose cardboard or a piece of dry flat cake (Figure 1). To impart porosity and nanoscale dimensions to the implantable particles, Dr. Sergey Litvinov produced a special biopolymer grid (alginate or collagen) attached to ordered nanocrystal chains of the biocompatible and water-insoluble substance, which served as a feed for cells that provide natural bone repair and regeneration. ${ }^{3}$ It is well-known that a human body does not accept many foreign substances because they cause perturbation at the cellular level. However, the effect from macrophages, whose role is to envelope foreign objects, ceases when implanted particles have nanoscale dimensions. The implantable material containing nanocrystal chains is thus well tolerated by the body. The LiTar material is an mixture of collagen (or calcium alginate polysaccharide protein) and 43-45 $\mathrm{nm}$ calcium hydroxo-phosphate crystals (hydroxylapatite) (Figure 2). Dr. Litvinov successfully achieved uniform distribution of the salt component between biopolymer fibers and determined optimum fiber/salt ratio. ${ }^{4}$

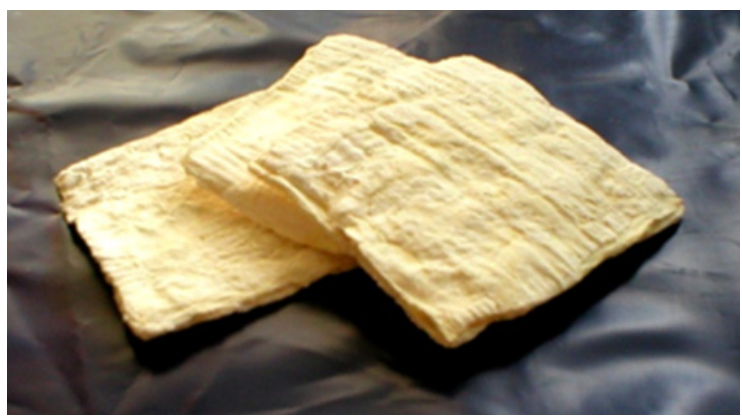

Figure I LitAr implantation material.

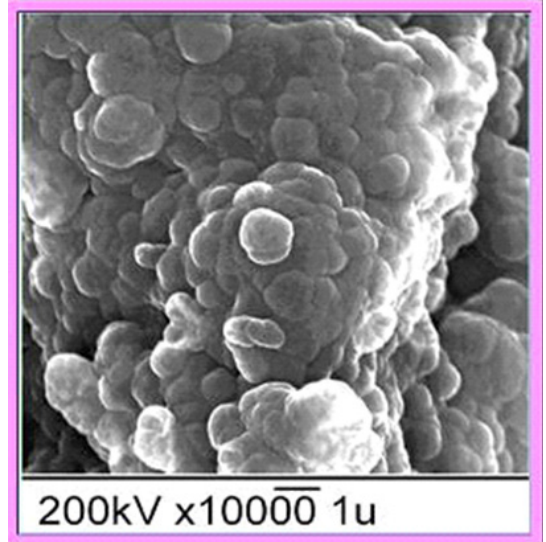

LiTar

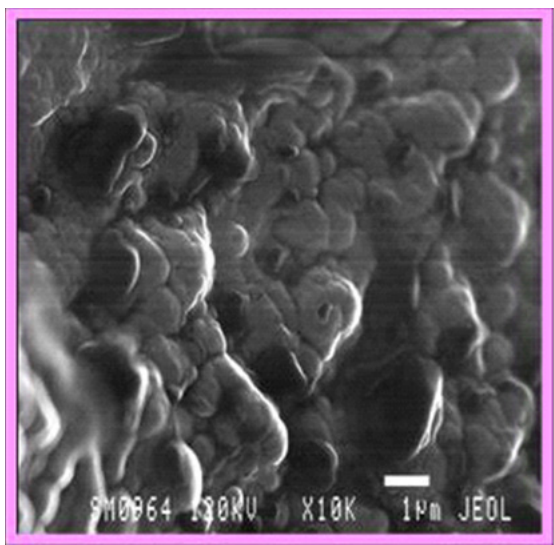

Bone tissue

Figure 2 The average sizes of apatite crystals in the material LitAr (44 nm) and bone tissue $(37 \mathrm{~nm})$ have similar values: It is precisely because of this LitAr acts on the body potent cells, providing complete bone tissue regeneration in traumatology and orthopedics without any possible negative rejection. 
The unique feature of the LitAr material is the stimulation of slightly differentiated stem cells in-vivo which, at the time of LitAr's material development, was not attained by anyone in the world. ${ }^{5}$ www.Lit-Ar.ru presents details of key differences between LitAr and its foreign analogs. Clinical testing which validated utility of Dr. Litvinov's technology were carried out by Prof. Vladimir Belokonev, Dr. Alexander Kosulin, a cranioplasty surgeon and Dr. Oleg Nikiforov, a thoracic surgeon. In 1994, the first operation was successfully conducted by Dr. Michail Babkov, assistant professor under the Prof. Alexander Krasnov direction. Figure 3 demonstrates the Litar application in the restored bone tissue. Another consequences of this fracture, such as Concussion or Stroke are not under consideration here. The first operations confirmed that LitAr provided regeneration of the lost part of the tissue in accordance with the normal anatomical structure in this part of the body. The Litar implementation is possible by two primary methods:

1) A surgery where a piece of material is inserted into patient's bone cavity.

2) Injection when the LitAr material is administered as a suspension in a physiological solution. ${ }^{6}$

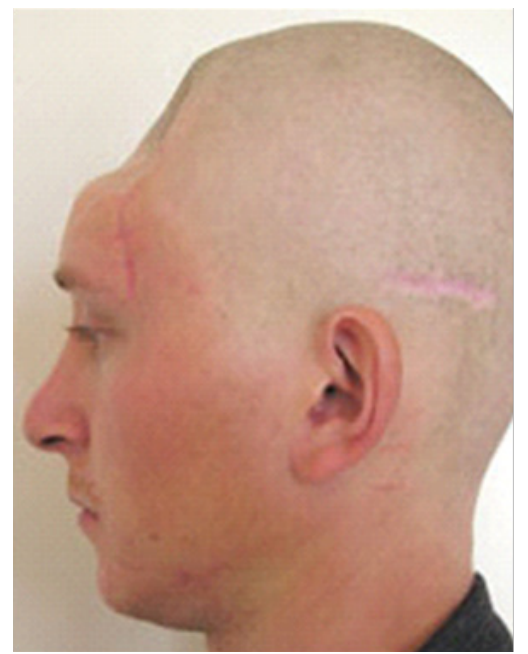

Before the operation

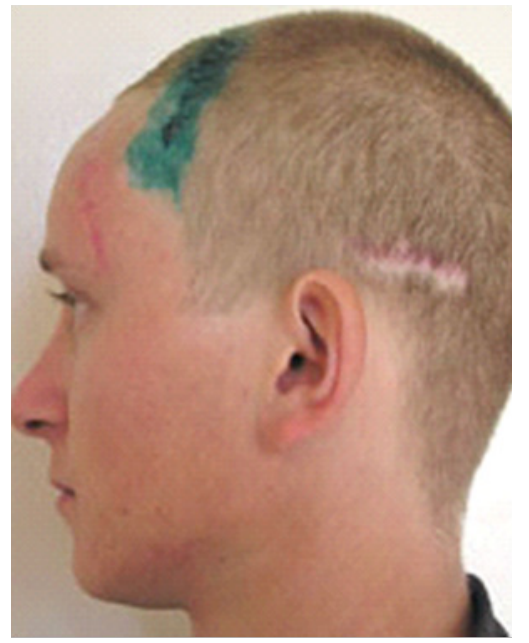

After it

Figure 3 Demonstrate the Litar application in the restored bone tissue.
As an example, the osteoplastic and collagen-apatite composite based on LitAr technology was successfully applied in treating of a patient with a large forehead fracture (Figure 4). In 2001, the LitAr material was used for the first time to be inserted into the thoracoabdominal fistula (Dr. Alexander Kulikov, Russia's Togliatti city). More recently, the LitAr material provided regeneration of not only bone, but also kidneys and liver tissues. The LitAr composite implementation against heart attacks have already become possible, however cardiac surgeons have little knowledge of the technology. Today Samara city has all necessary conditions for establishing of the International Medical Center on the model similar to the Ilizarov's Center in Kurgan city. LitAr material has obtained all of the required regulatory certification documents. Significant clinical benefit following the use of Litar material has been demonstrated. However, Russia's medical officials took the position of indifferent observers. On behalf of the International Academy of Sciences on Informational Safety, authors addressed the former Samara region authorities with a proposal that can accelerate commercialization of the LitAr material. But we only received very limited interest from government officials. This provides an example of extremely slow translation of biomedical technology which has already shown clinical benefits. Recently, health authorities in France's Montpellier city conducted research on the cartilage regeneration by LitAr material. There is likelihood that LitAr innovation will become commercialization abroad sooner that in Russia. If this is to happen, Russia's patients and the government will be paying a premium for the technology that its scientists invented.
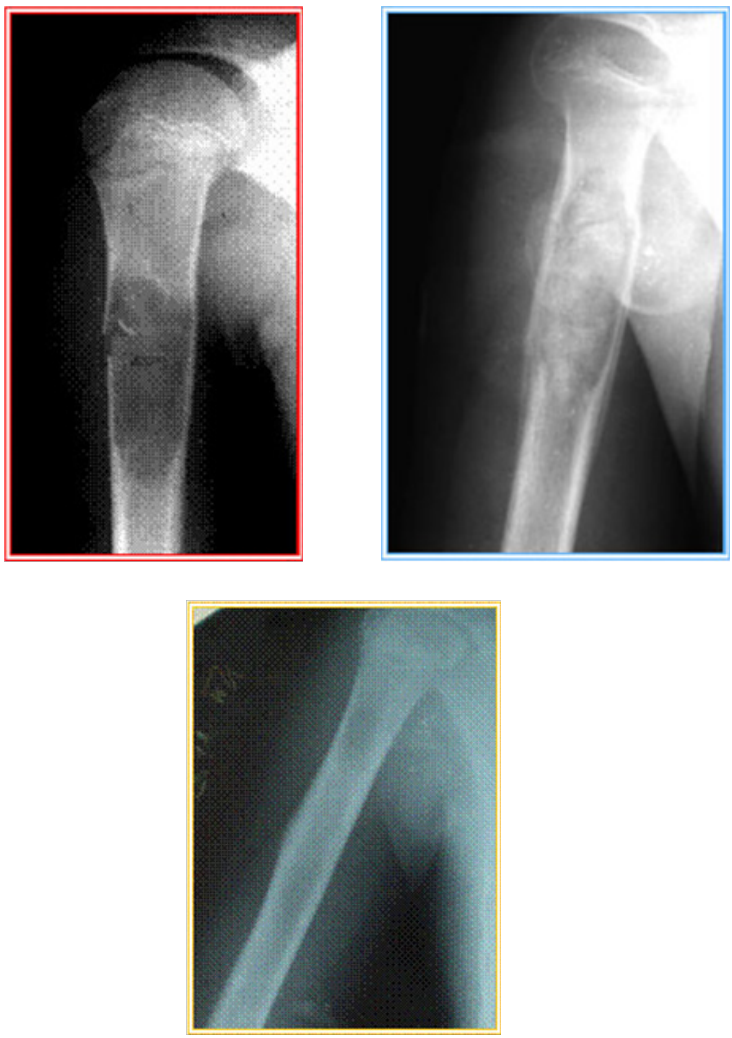

Figure 4 Patient M's X-ray before filling in the defect and I, and I 2 months after the operation. On the defect spot, one can barely see the unclear blur of the restored bone tissue.

Aleksey Valyaev is a co-author of a research which investigated that use of stimulus-responsive molecules grafted onto surfaces for biological sensing applications. ${ }^{7}$ Results demonstrated proof of 
principle for using microcantilevers coated with stimulus-responsive molecules for bio- detection of changes in solution $\mathrm{pH}$, temperature, and ionic strength in microliter volumes. With additional development, this biosensing technology can be potentially used in medical diagnostic applications e.g., where microfluidic devices developed for sampling of biological fluids can be actuated by local changes in solution $\mathrm{pH}$ or temperature. However, commercial translation of this technology would require significant financing and experienced management. He is also an inventor of an eye apparatus for use by people with dry eye disease. The apparatus enables combining several treatment modalities including the use of microparticle spray to improve therapeutic benefit for dry eye patients. Dry eye disease (DED) is a highly prevalent disorder resulting in hyperosmolarity of the tear film and inflammation of the ocular surface. According to the American Academy of Ophthalmology, more than three million women older than 50 suffer from dry eye syndrome in the United States. DED cause disruption of daily activities, reduction of work productivity due to recurrent blurred vision and ocular discomfort, and the overall burden of the disease for the US healthcare system is estimated at 4 billion. Because of relatively low regulatory constraints and significant market potential, with additional support the invented apparatus can be potentially commercialized in less than 5 years. We would also like to note here some thematic achievements of the Russian dentists. In State Medical University in Tver city, under Prof. Valery Strelnikov direction the long-term systematic researches are conducted on the use of biochemical markers of ostaclenogenesis in dental implantation and directional bone regeneration. ${ }^{8,9}$

Currently, indications for the study of markers of bone metabolism are the following diseases: postmenopausal and senile osteoporosis; glucocorticoid-induced osteoporosis; diseases with a local increase in resorptive activity; monitoring of osteoprotegerin therapy; arthritis; oncological diseases. The main purpose was to study the serum osteoprotegerin and s-rank levels in dental patients with different results of bone implant integration using bone grafts of xenogenic origin. 63 patients were examined: 41 women and 22 men aged 40 to 67 years. The general condition of the body was assessed on the basis of the collection of anamnesis of life, the conclusion of the therapist and blood test data. Osteoprotegerin and s rank - 1 osteoclastogenesis markers were studied in all patients, who were selected for the study with their voluntary consent. It is necessary to note the presence of common diseases, such as osteoporosis, diabetes mellitus, impaired immune system, in some patients. All of the above diseases are established by the thematic specialists and are not absolute contraindications to dental implantation. Thus, thanks to the present study, it is possible to expand the spectrum of absolute contraindications to dental implantation.

\section{Conclusion}

The above demonstrated examples suggest that biomedical innovators in Russia need much longer time to successfully commercialize new technologies than innovators in the US and Europe. The technology commercialization examples presented here suggest the following typical reasons that may inhibit commercialization of biomedical innovations in Russia:

a) The system of government and private support towards commercialization of biomedical and health-related innovation is not sufficiently established.

b) The Russia's largest scientific centers are weakly connected with manufacturing companies and hospital system. c) Highly productive innovators are rarely appointed to managerial roles or decision-making positions.

d) Bureaucracy hinders those changes to our education system that are required by constantly evolving innovation industry. The unification of the Russian Academy of Sciences with the Academies of Medical and Agricultural Sciences has significantly complicated and confused the situation with decision making in Russia's innovation ecosystem.

Because of the bureaucracy in the Russian Academy of Sciences many talented people are forced to leave their research positions in order to advance professionally. Many young innovators in Russia are not promoted due to protectionism by long-serving scientific authorities. To circumvent the issue some scientists have recently proposed to hold re-election and rotation of academics every 4 years, taking into account their actual scientific achievements for each reporting period. Slow reforms and misjudged decisions in regards to managing Rusia's science and technology transfer. The establishment of the Federal Agency of Scientific Organizations (FASO) along with the unification of the Russian Academy of Sciences with the Russian Academy of Medical Sciences and the Academy of Agricultural Sciences have significantly complicated a number of issues and caused additional expenses of the state budget towards newly elected academicians. The confrontation between the Russian Academy of Sciences and the FASO has significantly inhibited the development of scientific breakthroughs and technology translation. In one example, FASO has introduced the controversial plan concerning the number of articles that have to be prepared by RAS staff for years to come. Some of the existing FASO issues have been analyzed in details and substantiated by the Russian Academician of Sergei Stishov. He considers that the FASO wants to turn the Russian Scientists into Non-Stop Machines that Produce Unnecessary Articles. To mitigate consequences from past mistakes and overcome existing innovation challenges, Russia needs science and technology translation programs that are not influenced by slowly moving bureaucratic processes.

\section{Proposed steps}

We propose several alternative concerning measures and systemforming elements to accelerate technology development and translation. These include the following:

1. Perform fast and objective certification of scientific personnel without long-term clearance procedure to get postdoctoral degrees;

2. Objectively and continuously evaluate performance and productivity of scientists;

3. Take active measures to remove bureaucratic obstacles that inhibit scientific innovation and translation;

4. Industry participation in commercialization should prevail over misguided decisions of administrative or government officials;

5. Establish mechanisms that reward prolific inventors. Make progress in technological innovation and commercialization as part of evaluation of regional leaders and officials.

6. Nobel laureate Andrei Geim said that money alone cannot solve scientific problems. Yet each dollar invested in a successfully operating laboratory will be paid off faster and give a better result than substantial capital injections into large innovation centers like Skolkovo and Rosnano, that did not have their own established scientific schools. 
7. Strengthening Russia's system of intellectual property protection that would encourage more scientists to disclose and protect their inventions;

8. Because successful commercialization of bio and nanotechnologies requires wide ranging support and participation, we need to develop a comprehensive plan which includes inputs from scientific community, industry and government representatives.

9. The using of numerous examples on LitAr successful application, presented in ${ }^{5}$ and in according to Prof. Sergey Petrov opinion, it is most preferable to create a specialized center for the integration of 3 above mentioned RF technologies in treating severe injuries most preferably in the Samara region with real active help from Prof. Sergey Litvinov, constantly living in Samara.

Therefore, it would be logical to combine such technologies in those operating medical centers, hospitals and clinics, that have already successfully applied them. With RHCS financial support it would be advisable to create the thematic departments in all RF major operating surgical centers with the thematic training courses, based on Samara clinics. This is the essence of the implementation problem with creating financial support. It is especially difficult to organize for the Russian provincial doctors and the thematic scientists. The problems analysis presented by us is a necessary step. We hope that the scientific community efforts will help to change the attitude to innovations for the better in the interests of the whole humankind.

\section{Acknowledgments}

None.

\section{Conflicts of interest}

Author declares that there is no conflict of interest.

\section{References}

1. Litvinov S, Ershov Y, Krasnov AF. Biotransformation of Synthetic Implants in the Course of Making Bone Tissue Prosthetics. Canadian Journal of Phisiology and Pharmacology. 1994:297.

2. Litvinov S, Krasnov A, Ershov Yu A. Specific Features of Bone Tissue Regeneration after Replacement of the defect with a Synthetic Implant. Bulletin of Experimental Biology and Medicine. 1995;119(4):422-425.

3. Ershov I, Litvinov S. The Rate of Solution of Hydroxyapatite Reinforced with Collagen as the Criterion of Polymer Implant Materials Quality. International Journal of Polymaric Materials. 1995;28(1-4):83-89.

4. Litvinov SD. Biological and clinical study kinetics of biodegrading apatite- collagen implant for substituting bone tissue defects. European Journal of DRUG METABOLISM and pharmacokinetics. 1998;28(2):346-349.

5. Petrov SV. Experience of introducing the material "Litar" to recover from severe injuries. Bulletin of the medical institute. 2017;5:3-11.

6. Volynkina MV. The problem of introducing scientific and technological achievements: the historical view.

7. Valiaev A, Abu-Lail N, Lim DW, et al. Micro-Cantilever Sensing and Actuation with End-Grafted Stimulus-Responsive Elastin-Like Polypeptides. Langmuir. 2007;23(1):339-344.

8. Strelnikov VN. Changes in indicators of osteocalcin, the bone isoenzyme of alkaline phosphatase and cathepsin $\mathrm{K}$, in the serum of dental patients with comorbidities. Periodontology. 2014;70:20-23.

9. Strelnikov VN. Prediction of the results of orthopedic treatment of patients with loss of teeth on artificial supports. Tver city. State Medical University; 2014. p. 231 